\title{
Mobile Stroke Unit Hits the Road in Houston
}

\author{
James C Grotta, MD \\ Director of Stroke Research, Clinical Innovation and Research Institute, Memorial Hermann-Texas Medical Center; Director, Mobile Stroke \\ Unit Consortium, Houston, Texas, US
}

\begin{abstract}
After 1 year of preparation, the nation's first Mobile Stroke Unit (MSU) delivering acute stroke treatment with tissue plasminogen activator (tPA) in the prehospital setting was launched in mid 2014. The unit is being operated as part of a clinical trial comparing MSU management to standard management to determine how much faster patients can be treated, how much better patients do if treated in the first hour after symptom onset than if treated later, if the physician on board the MSU can be replaced by a remote physician via telemedicine, and the costs and quality-adjusted life years saved by the MSU approach. We are treating on average over two patients per week with intravenous tPA, with more than $30 \%$ treated within the first hour of symptom onset.
\end{abstract}

\section{Keywords}

Stroke, tissue plasminogen activator, thrombolysis, ambulance, EMS, prehospital, computed tomography, telemedicine

Disclosure: The Mobile Stroke Unit (MSU) and research project have been funded by donations from local philanthropists, as well as grants from Covidien, Frazer Ltd, and Genentech. James C Grotta, MD, has no conflicts of interest to declare. No funding was received for the publication of this article.

open Access: This article is published under the Creative Commons Attribution Noncommercial License, which permits any noncommercial use, distribution, adaptation, and reproduction provided the original author(s) and source are given appropriate credit.

Received: December 27, 2015 Accepted: January 21, 2015 Citation: US Neurology, 2015;11(1):59-61 DOI: 10.17925/USN.2015.11.01.59

Correspondence: James C Grotta, MD, Director of Stroke Research, Clinical Innovation and Research Institute, Memorial Hermann Hospital-TMC, Houston, TX 77030, US.

E: yvette.sanders@uth.tmc.edu

The nation's first Mobile Stroke Unit (MSU) was launched in February, 2014 by James Grotta, MD, and colleagues at the Texas Medical Center in Houston. The concept, pioneered by Marie Curie when she loaded an X-ray machine onto an ambulance during World War I, is logical: to take emergency care to the patient in order to allow accurate diagnosis and urgent treatment earlier when it may do more good. Now 100 years later, instead of a physicist and $\mathrm{X}$-ray machine, it is a vascular neurologist (VN) and computed tomography (CT) scanner.

Time is particularly important in acute stroke therapy. Data from primates and rodents show that if recanalization occurs during the first hour after arterial occlusion, cerebral infarction might be completely avoided except in the 'core' of distal carotid or proximal middle cerebral artery occlusions. ${ }^{1}$ Surprisingly, the initial National Institute of Neurological Disorders and Stroke (NINDS) tissue plasminogen activator (tPA) study results did not seem to reflect this relationship. But once it was recognized that patients with the most severe strokes presented to the emergency department (ED) earlier and stroke severity was factored into the calculation, it became clear that the sooner tPA was given, the better the clinical response to tPA. ${ }^{2}$ The same relationship exists with endovascular mechanical reperfusion, ${ }^{3}$ and probably efforts to reduce bleeding by blood pressure lowering or coagulopathy reversal after intracerebral hemorrhage.

While the use of tPA is increasing (now 5-10\% of all stroke patients; 10-20 \% in most stroke centers), treatment usually occurs $2-5$ hours after symptom onset even if patients or bystanders do the right thing and call $911 .{ }^{4}$ In the NINDS study, none of the 302 patients who were randomized within the first 90 minutes actually were treated within the first 60 minutes from onset. ${ }^{5}$ Current national databases show that less than $5 \%$ of treatments occur in the first hour. ${ }^{4}$ The delay is multifactorial and includes delay in calling 911, mobilizing emergency medical services (EMS) and transporting patients to the right ED, and, finally, the ED door to needle time, which stubbornly averages 50-60 minutes in even our best stroke centers, largely taken up by time to obtain the CT scan and having the decision-maker (usually the neurologist on call) look at it and make a decision after weighing all the variables.

The MSU cuts out the entire door-to-needle-time delay by putting the CT scanner and decision-maker on the ambulance. The initial studies conducted in Germany and published over the past 2 years demonstrated anywhere from 25-80 minute time savings, and over $30 \%$ treated within the first hour after symptom onset, without excess complications. ${ }^{6-8}$ In 2013, after visiting Drs Fassbender and Audebert who pioneered the MSU concept in Germany, Dr Grotta decided to implement an MSU in Houston.

There were three basic principles that guided how this project would be carried out. First, we understood that no funding was available from UT Medical School, Memorial Hermann Hospital, or the City of Houston to underwrite this project. Second, we needed to operate within the current EMS transportation and triage system, which delivered acute stroke patients 
within 30 minutes drive from the Texas Medical Center equally to one of three comprehensive stroke centers (CSCS). Third, our goal was to determine if the MSU is a practical strategy for speeding stroke care that could have a meaningful impact on stroke outcome nationwide.

To make this determination, we decided to carry out a clinical trial to answer three questions: 1. How much faster could we treat patients and how much better outcome would result? 2. Could we replace the VN on board the MSU by a remote VN using telemedicine (TM)? 3. What would be the balance between the benefits and the costs of implementing and deploying the MSU? The Benefits of Stroke Treatment Delivered Using a Mobile Stroke Unit Compared to Standard Management by Emergency Medical Services (BEST-MSU) study was approved by the UT-Houston IRB on January 11, 2013 to address these aims and is registered with clinicaltrials.gov (NCT02190500).

We began fundraising in March 2013 eventually raising \$1.8M largely from donations by generous Houston citizens and businesses, and grants from pharmaceutical and device manufacturers. Two critical early donors deserving particular recognition were Frazer Ltd from Bellaire, Texas who donated the ambulance 'box', and James ('Mattress Mack') McIngvale. After purchasing the Neurologica CereTom CT scanner, many steps then went into building, licensing, inspecting, insuring, equipping, and staffing the MSU. This required the $100 \%$ effort of a multitalented Project Manager, Stephanie Parker RN. Frazer modified a standard 12-foot ambulance used by the Houston Fire Department to accommodate the CT scanner. Extensive radiation safety inspection, and licensing by both state and city authorities as an ambulance under the umbrella of Memorial Hermann Life Flight required the cooperation of administrators at UT Medical school and Memorial Hermann. Leadership of the stroke teams at Baylor St Lukes, Memorial Hermann, and The Methodist Hospitals, along with Houston Fire Department EMS and the MSU team formed the Houston Mobile Stroke Unit Consortium with a Steering Committee that oversees the entire project.

The MSU is staffed by a VN, a Registered Nurse (both with advanced cardiovascular life support [ACLS] training), a full-time licensed radiology CT tech, and an off-duty EMS paramedic, and also has remote access to a second VN at UT Medical School via TM. In addition to standard emergency equipment and the CT scanner, the MSU also has point-of-care laboratory capability. An MSU office was rented at the UT Professional Building, 6410 Fannin St where we also rent a parking space with power supply for the MSU.

Once the MSU was delivered by Frazer, we developed onboard protocols for all advanced life support scenarios, and carried out extensive inservicing of EMS personnel in order to integrate the MSU into the daily operations of preclinical care in Houston, Bellaire, and West University. A communications system was devised where the MSU would be notified by the dispatch centers of each of these three cities after a 911 call suggestive of a stroke, or if a paramedic requested MSU dispatch after recognizing a stroke patient on-scene. The MSU primarily responds to calls within a 5-mile radius of the MSU offices, but can also rendezvous with EMS squads bringing patients to the TMC from a greater distance. The design is for the MSU to meet up with the 'regular' EMS squad without delaying or altering their routine prehospital management. The MSU team responds to EMS calls every day from 8 am to 6 pm, except designated holiday weeks.

Upon receiving a call, the MSU travels to the site of the call or rendezvous with EMS and evaluates the patient. If the patient meets the inclusion criteria (symptom onset within 4.5 hours, and meeting published criteria for tPA treatment pending CT scan and baseline labs), they are enrolled into the study and moved into the MSU. If after CT scan and point-of-care lab testing on the MSU, the patient still fulfills criteria for TPA according to the on-site VN (the patient is simultaneously evaluated via TM with the remote $\mathrm{VN}$ making an independent decision), they are immediately given TPA and transported to one of our three CSCS. If the patient does not meet tPA criteria, they are managed as per best practice for their diagnosis en route to the CSC. This may include blood pressure lowering or coagulopathy reversal in the case of intracerebral hemorrhage.

The MSU went into service on May 14, 2014. According to a prespecified plan, the MSU was activated from 8 am to 6 pm for an 8-week 'run-in' phase to test the communication system, rehearse interactions between EMS, the MSU team, and the remote TM VN, test out the case report forms, and confirm projected MSU activity and treatment rates.

During the 'run-in' phase, the MSU was dispatched by the dispatch center on 130 occasions, or roughly 2.7 per day. The MSU was 'disregarded' en route to the scene for 41 of these dispatches when it was determined by either the first responders or paramedics to not fit study criteria. For another 65 of these dispatches, the MSU team arrived on scene, assessed the patient, and determined the patient did not qualify. These 106 patients were considered 'screen failures.' They were transported as per EMS routine and no further data were obtained.

Twenty-four patients met criteria for enrollment (symptom onset within 4.5 hours, and meeting published criteria for tPA treatment pending CT scan and baseline labs). Eleven of these patients were not treated. Four had primary intracerebral hemorrhage and had their blood pressure acutely lowered according to our current standard of care protocol. Three had seizures on board the MSU, which were thought to be the cause of their presentation, and which were treated on board the MSU. Two patients improved to the point where the MSU staff determined that TPA was not indicated. The time of onset could not be confidently determined in one patient, and one had a subdural hematoma. Thirteen patients were treated with tPA on the MSU: four (31\%) between 0-60 minutes of onset, four (31\%) between 61-80 minutes from onset, and five (38\%) between 81-270 minutes of onset. Average on-scene time from MSU arrival to tPA bolus was 24 minutes (range 12-53) in the 13 treated patients. There were no hemorrhagic or other complications and no malfunctions of the CT scanner or MSU. The intravenous infusion pump malfunctioned on one patient, and the i-STAT device malfunctioned because of heat on one occasion. TM assessment of the patient was carried out successfully in all 11 cases in which remote assessment was attempted, and agreement between the remote and on-site VN was $91 \%$. Three of our 13 patients had endovascular treatment with onset to groin times of 224, 140, and 150 minutes.

On August 19, 2014 we began randomizing into the BEST-MSU study. Since then our IPA treatment rate on the MSU continues to average two to three patients per week, with over $40 \%$ treated within the first 60 minutes of 
stroke symptom onset. In order to carry out the BEST-MSU study, on $50 \%$ of weeks by blocked randomization, the nurse meets the patient without the MSU, determines eligibility by the same criteria, but the patient is transported and managed per current EMS routine. Informed consent is obtained from the patient or next of kin at the CSC after all acute stroke care is complete to obtain follow-up data at 1, 3, 6, and 12 months in 248 patients to answer the three aims. The Data Management and Health Economics Center is at the UT School of Public Health.

In summary, the Houston Mobile Stroke Unit is in full-time operation and should answer important questions about the feasibility of this approach to acute stroke care through the BEST-MSU study. We anticipate it will take 4-5 years to complete this study. Additional funding is needed and is being sought from granting agencies and donors. Other MSU sites in other cities are in various stages of development to partner with us to answer the aims of the BEST-MSU study more quickly. If we are successful, eventually we envision 4-5 MSUs strategically deployed and embedded within the Houston Fire Department EMS fleet (and proportionate coverage throughout the rest of the country), with TM coverage and three persons on board: two paramedics and a third person to interact with TM and cross-trained to carry out the CT scanning. The costs of building and staffing these MSUs would be amortized by higher reimbursement from payers (insurers) justified by the cost savings resulting from better outcomes and reduced long-term care requirements.
1. Jones TH, Morawetz RB, Crowell RM, et al., Thresholds of focal cerebral ischemia in awake monkeys, J Neurosurg, 1981;54:773-82.

2. Marler JR, Tilley BC, Lu M, et al., Early stroke treatment associated with better outcome: the NINDS rt-PA stroke study, Neurology, 2000;55:1649-55.

3. Khatri P, Yeatts SD, Mazighi M, et al., Time to angiographic reperfusion and clinical outcome after acute ischaemic stroke: an analysis of data from the Interventional Management of Stroke (IMS III) phase 3 trial, Lancet Neurol, 2014;13:567-74.
4. Fonarow GC, Smith EE, Saver JL, et al., Timeliness of tissue-type plasminogen activator therapy in acute ischemic stroke: patient characteristics, hospital factors, and outcomes associated with door-to-needle times within 60 minutes, Circulation, $2011 ; 123: 750-8$

5. No authors listed, Tissue plasminogen activator for acute ischemic stroke. The National Institute of Neurological Disorders and Stroke rt-PA Stroke Study Group, N Eng/ I Med, 1995;333:1581-7.

6. Walter S, Kostopoulos P, Haass A, et al., Diagnosis and treatment of patients with stroke in a mobile stroke unit versus in hospital: a randomised controlled trial, Lancet Neurol, 2012;11:397-404.

7. Ebinger $M$, Winter $B$, Wendt $M$, et al., Effect of the use of ambulance-based thrombolysis on time to thrombolysis in acute ischemic stroke: a randomized clinical trial, JAMA 2014:311:1622-31

8. Ebinger $M$, Kunz $A$, Wendt $M$, et al., Effects of golden hourthrombolysis. A prehospital acute neurological treatment and optimization of medical care in stroke (PHANTOM-S) substudy, JAMA Neurol, 2015;72:25-30. 